\title{
Richard Wiegandt
}

On the structure of lower radical semigroups

Czechoslovak Mathematical Journal, Vol. 22 (1972), No. 1, 1-6

Persistent URL: http://dml.cz/dmlcz/101074

\section{Terms of use:}

(C) Institute of Mathematics AS CR, 1972

Institute of Mathematics of the Czech Academy of Sciences provides access to digitized documents strictly for personal use. Each copy of any part of this document must contain these Terms of use.






\title{
ON THE STRUCTURE OF LOWER RADICAL SEMIGROUPS
}

\author{
RichaRd WIEGANDT, Budapest
}

(Received November 22, 1969)

1.

The basic idea of this paper is due to the fact discovered in the recent categorytheoretically investigations of [12], that the Wedderburn-Artinian decomposition theory of rings and STEINFELD's [7] analogous results for semigroups are categorically duals of each other. The exquisite results of ReEs [5], Schwarz [6] and STEINFELD $[7]$, [8] establish satisfactory characterizations of semigroups being unions of complete 0-simple subsemigroups. Up to this time such "well-behaved" semigroups were considered and treated as semisimple semigroups, and indeed they are semisimple with respect to the nil radical property [6], but these semigroups do not satisfy those properties what a semisimple class ought to satisfy. Since the radical and semisimple properties are categorically dual notions (cf. [10]), so by the duality mentioned before it is reasonable to try to characterize all the semigroups of the smallest radical class which contains these well-behaved semigroups. Thus the well-behaved semigroups will be considered as radical semigroups according to an appropriate radical property. Using the methods of the recent ring-theoretical developments, namely those of Suliński-ANDERSON-Divinsky [9], Armendariz-Leavitt [1] and Watters [11], we shall construct the lower radical class determined by a class of simple idempotent semigroups with zero. It will turn out that this lower radical class is always hereditary, further the lower radical semigroups will be classified in Theorem 2. Thus for instance, if the lower radical class is determined by the class of completely 0 -simple semigroups, then to any radical semigroup $S$ there belong an ordinal number depending on $S$ and a strictly ascending chain $0=I_{0} \subset \ldots \subset I_{\zeta}=S$ of twosided ideals of $S$ such that every Rees factor semigroup $I_{\lambda+1} / I_{\lambda}(0 \leqq \lambda<\zeta)$ is the 0 -disjoint union (i.e. the 0 -direct union) of completely 0 -simple subsemigroups (and the complete 0 -simple semigroups are isomorphic to regular Rees matrix semigroups). 
For standard semigroup-theoretical definitions and results we refer to [2] or [4]. In the following a semigroup will mean always a semigroup with zero. If $I$ is an ideal (always two-sided) of a semigroup $S$, then $S / I$ will denote the Rees factor semigroup of $S$, i.e. the semigroup $\left.S^{\prime}=O \cup(S \backslash I]\right)$ and if $a b=c \in I$ for any two elements $a, b \in S$, then we define $a b=0$ in $S^{\prime}$. A semigroup and an ideal belonging to a certain class $\mathscr{C}$ will be called a $\mathscr{C}$-semigroup and a $\mathscr{C}$-ideal, respectively. We shall say that the class $\mathscr{C}$ is homomorphically closed if every Rees factor semigroup of a $\mathscr{C}$-semigroup is again a $\mathscr{C}$-semigroup. The class $\mathscr{C}$ is hereditary, if every ideal of a $\mathscr{C}$-semigroup belongs to the class $\mathscr{C}$.

A class $\mathscr{R}$ of semigroups is said to define a radical property in the sense of KurošAmitsur, if the following conditions hold:

(A) $\mathscr{R}$ is homomorphically closed.

(B) Every semigroup $S$ contains an $\mathscr{R}$-ideal $\mathscr{R}(S)$ which contains every other $\mathscr{R}$-ideal of $\mathscr{S}$.

(C) The Rees factor semigroup $S / \mathscr{R}(S)$ does not contain any non-zero $\mathscr{R}$-ideal.

In this case the class $\mathscr{R}$ is called a radical class. $\mathscr{R}(S)$ is called the $\mathscr{R}$-radical of $S$. A semigroup $S$ is called an $\mathscr{R}$-radical semigroup, if it belongs to the class $\mathscr{R}$, that is $S=\mathscr{R}(S)$.

Let $\mathscr{M}$ be a class of semigroups. A radical class $\mathscr{L} \mathscr{M}$ is called the lower radical class of $\mathscr{M}$, if $\mathscr{L} \mathscr{M}$ is a radical class, and $\mathscr{L} \mathscr{M}$ is contained in every radical class which contains $\mathscr{M}$. A lower radical semigroup is a semigroup of a lower radical class.

Proposition 1. A class $\mathscr{R}$ of semigroups defines a radical property if and only if (A) $\mathscr{R}$ is homomorphically closed.

(D) If every non-zero Rees factor semigroup of a semigroup $S$ contains a nonzero $\mathscr{R}$-ideal, then $S$ is an $\mathscr{R}$-semigroup.

Proof. The proof is analogous to the ring-theoretical one (cf. Divinsky [3] Theorem 1) For the sake of completeness we give the detailed proof.

Assume that $\mathscr{R}$ is a radical class. It is sufficient to see that (B) and (C) implies (D). Let $S$ be a semigroup which is not an $\mathscr{R}$-semigroup. Then by (B), it has an $\mathscr{R}$-radical $\mathscr{R}(S) \neq S$. Then by $(\mathrm{C})$ the non-zero Rees factor semigroup $S / \mathscr{R}(S)$ does not contain a non-zero $\mathscr{R}$-ideal. This established (D).

To prove the converse, assume $\mathscr{R}$ satisfies the conditions (A) and (D). Let $J$ be the set-theoretical union of all $\mathscr{R}$-ideals of a semigroup $S$. If $J=0$, then it is an $\mathscr{R}$-ideal. If $J \neq 0$, then let $K$ be an arbitrary proper ideal of $J$. Since $K \subset J$, therefore there must be an ideal $L$ of $S$ such that $L$ is not contained in $K$. By the second isomorphism theorem $(L \cup K) / K \cong L /(L \cap K)$ holds. Since by $(\mathrm{A}) L /(L \cap K)$ belongs to $\mathscr{R},:$ so $(L \cup K) / K$ is a non-zero $\mathscr{R}$-ideal of $J / K$. Thus by (D) $J$ is an $\mathscr{R}$-ideal, and condition (B) is satisfied. 
Consider now the $\mathscr{R}$-radical $J=\mathscr{R}(S)$ of $S$, and the Rees factor semigroup $S / J$. Suppose that $S / J$ contains a non-zero $\mathscr{R}$-ideal $M / J$. Then $M$ is an ideal of $S$. Let $M / N$ be any non-zero Rees factor semigroup of $M$. If $J \subseteq N$, then by (A) we have

$$
0 \neq M / N \cong \frac{M / J}{N / J} \in \mathscr{R} .
$$

If $J \notin N$, then $N \cap J$ is an ideal of $M$ and $0 \neq(N \cup J) / N \cong J /(N \cap J)$ holds. Since by (A) $J /(N \cap J)$ belongs to $\mathscr{R}$, so $(N \cup J) / N$ is a non-zero $\mathscr{R}$-ideal of $M / N$. Thus (D) implies $M \in \mathscr{R}$, and so $M \subseteq J$ follows, contradicting the assumption $M / J \neq 0$.

Any homomorphically closed class $\mathscr{M}$ of semigroup defines a radical class $\mathscr{L} \mathscr{M}$ as follows (cf. [9]). ${ }^{1}$ )

Proposition 2. Let $\mathscr{M}$ be a homomorphically closed class of semigroups. Define $\mathscr{M}=\mathscr{M}_{1}$. Assuming that $\mathscr{M}_{\alpha}$ has been defined for every ordinal number $\alpha<\beta$, we define $\mathscr{M}_{\beta}$ to be the class of all semigroups $S$ such that every non-zero Rees factor semigroup of $S$ contains a non-zero ideal I which is in $\mathscr{M}_{\alpha}$ for some $\alpha<\beta$. Then $\mathscr{L} \mathscr{M}=\bigcup_{\alpha} \mathscr{M}_{\alpha}$ is a radical class.

Proof. Every class $\mathscr{M}_{\beta}$ is homomorphically closed. In the case $\beta=1$ the statement is trivial. If $\beta>1$, then consider a non-zero Rees factor semigroup $S^{\prime}=S / I$. Since every Rees factor semigroup $S^{\prime \prime}=S^{\prime} \mid J^{\prime}$ is a Rees factor semigroup

$$
S^{\prime \prime}=\frac{S / I}{J / I},
$$

so $S^{\prime \prime}$ contains a non-zero ideal belonging to $\mathscr{M}_{\alpha}$ for some $\alpha<\beta$. Thus $S^{\prime}$ belongs to $\mathscr{M}_{\beta}$.

This proves that also $\mathscr{L} \mathscr{M}$ is homomorphically closed, and so condition (A) is fulfilled.

In order to prove (D) let us observe that obviously $\mathscr{M}_{\alpha} \subseteq \mathscr{M}_{\beta}$ holds for $\alpha \leqq \beta$. Let $S$ be a semigroup such that every non-zero Rees factor semigroup of $S$ contains a non-zero ideal $I_{\sigma}$ belonging to $\mathscr{L} \mathscr{M}$. Hence $I_{\sigma}$ belongs to a class $\mathscr{M}_{\alpha_{\sigma}}$ for some ordinal number $\alpha_{\sigma}$. If $\beta$ is an ordinal number not less than any of the $\alpha_{\sigma}$, then we have $S \in \mathscr{M}_{\beta+1} \subseteq \mathscr{L} \mathscr{M}$. This establishes property (D).

Proposition 3. (cf. [3] Lemma 5). $\mathscr{L} \mathscr{M}$ is the lower radical class determined by $\mathscr{M}$ i.e. if $\mathscr{R}$ is a radical class such that $\mathscr{M} \subseteq \mathscr{R}$, then $\mathscr{L} \mathscr{M} \subseteq \mathscr{R}$ follows.

Proof. We show by induction $\mathscr{M}_{\beta} \subseteq \mathscr{R}$ for every ordinal number $\beta$. For $\beta=1$ the statement $\mathscr{M}_{1}=\mathscr{M} \subseteq \mathscr{R}$ is trivially fulfilled. Take $\beta>1$, and assume $\mathscr{M}_{\alpha} \subseteq \mathscr{R}$ for

1) Added in proof (3 February 1972): In the meanwhile there appeared a paper on lower radicals of semigroups: F. A. Szász, On radicals of semigroups with zero, Proc. Japan Acad., 46 (1970), 595-598 (submitted for publication on 22 June, 1970). 
every $\alpha<\beta$. If $S^{\prime}$ is a non-zero Rees-factor semigroup of an arbitrary semigroup $S \in \mathscr{M}_{\beta}$, then $S^{\prime}$ contains a non-zero ideal $I \in \mathscr{M}_{\alpha} \subseteq \mathscr{R}$. Since $\mathscr{R}$ satisfies condition (D), therefore $S \in \mathscr{R}$, and also $\mathscr{M}_{\beta} \subseteq \mathscr{R}$ follow.

We need also the analoguous statement of ANDRUNAKIEVIČ' Lemma ([3] Lemma 71).

Proposition 4. If $K$ is an ideal of $I$ and $I$ is an ideal of $S$ and if $K^{*}$ is the ideal of $S$ generated by $K$, then $K^{* 3} \subseteq K$.

Proof.

$$
K^{* 3} \subseteq I K^{*} I=I(K \cup S K \cup K S \cup S K S) I \subseteq I K I \subseteq K
$$

Proposition 5 (cf. [9] Theorem 2). If $\mathscr{M}$ is a homomorphically closed, class of idempotent semigroups, then the process of constructing $\mathscr{L} \mathscr{M}$ terminates at $\mathscr{M}_{1}$ or $\mathscr{M}_{2}$, i.e. $\mathscr{L} \mathscr{M}=\mathscr{M}_{2}$, is valid.

Proof. Take $S \neq 0$ in $\mathscr{M}_{3}$. Then any non-zero Rees factor semigroups $S^{\prime}$ of $S$ contain a non-zero ideal $I$ which is in $\mathscr{M}_{2}$. Furthermore, every non-zero homomorphic image of $I$, and in particular $I$ itself must contain a non-zero ideal $K$ which is in $\mathscr{M}_{1}=\mathscr{M}$. Let $K^{*}$ be the ideal of $S$ generated by $K$. By Proposition 4 $K^{* 3} \subseteq K$ holds. Now $0 \neq K=K^{3} \subseteq K^{* 3} \subseteq K$ follows, because $\mathscr{M}$ consist of idempotent semigroups. Thus $S^{\prime}$ contains a non-zero ideal $K$ which is in $\mathscr{M}_{1}$, and therefore $S \in \mathscr{M}_{2}$ holds.

Proposition 6 (cf. [1] or [11]). If a homomorphically closed class $\mathscr{M}$ is hereditary, then $\mathscr{M}_{2}$ is also hereditary. Particularly, if $\mathscr{M}$ is a homomorphically closed hereditary class of idempotent semigroups, then also the lower radical class $\mathscr{L} \mathscr{M}$ is hereditary.

Proof. Let $S \in \mathscr{M}_{2}$, let $I$ be an ideal of $S$ and $K$ an ideal of $I$ properly contained in $I$. Since $S \in \mathscr{M}_{2}$, there is a non-zero ideal $J \in \mathscr{M}$ of $S$. We shall prove that also $I / K \neq 0$ contains a non-zero $\mathscr{M}$-ideal.

If $J \cap I \nsubseteq K$, then $((J \cap I) \cup K) / K$ is a non-zero ideal of $I / K$, and by the second isomorphism theorem $((J \cap I) \cup K) / K \cong(J \cap I) /(J \cap K)$ holds. Since $\mathscr{M}$ is a hereditary class, so the ideal $J \cap I$ of $I$ belongs to $\mathscr{M}$. Hence also its Rees factor semigroup $(J \cap I) /(J \cap K)$ belongs to $\mathscr{M}$. Thus we have proved that in the case $J \cap I \nsubseteq K$ the Rees factor semigroup $I / K$ contains a non-zero $\mathscr{M}$-ideal $((J \cap I) \cup K) / K$.

If $J \cap I \subseteq K$, then the set of ideals whose intersection with $I$ is contained in $K$ is non-empty. By Zorn's lemma this set has a maximal element, $L$ say. Since $I \cap L \subseteq$ $\subseteq K \subset I$, so $L$ is a proper ideal of $S$. Hence $S^{\prime}=S / L$ is a non-zero Rees factor semigroups and thus it contains a non-zero $\mathscr{M}$-ideal $N / L$. Since $I \cap L \subseteq K \cap N$, therefore by the second isomorphism theorem we have

$$
((I \cap N) \cup L) / L \cong(I \cap N) /(L \cap I \cap N)=(I \cap N) /(I \cap L),
$$


and the maximality of $L$ implies $(I \cap N) /(I \cap L) \neq 0$. Further $((I \cap N) \cup L) / L$ is an ideal of $N / L \in \mathscr{M}$ and $\mathscr{M}$ is hereditary, so also $((I \cap N) \cup L) / L \in \mathscr{M}$ is valid. Hence $(I \cap N) /(I \cap L)$ is a non-zero element of the class $\mathscr{M}$. On the other hand the second isomorphism theorem implies

$$
((I \cap N) \cup K) / K \cong(I \cap N) /(K \cap I \cap N)=(I \cap N) /(K \cap N)
$$

and so by $I \cap L \subseteq K \cap N$ we have that $(I \cap N) /(K \cap N)$ is a Rees factor semigroup of $(I \cap N) /(I \cap L) \in \mathscr{M}$. Hence $((I \cap N) \cup K) / K$ in a non-zero $\mathscr{M}$-ideal of $I / K$.

\section{3.}

Let $\mathscr{M}$ be a class consisting of all 0-simple idempotent semigroups and of all oneelement semigroups. Now $\mathscr{M}$ is homomorphically closed as well as hereditary. Propositions 5 and 6 infer immediately

Theorem 1. The lower radical class $\mathscr{L} \mathscr{M}$ is hereditary, moreover $\mathscr{L} \mathscr{M}=\mathscr{M}_{2}$ holds.

Theorem 1 yields a structure theorem according to the $\mathscr{L} \mathscr{M}$-radical semigroups (i.e. $\mathscr{L} \mathscr{M}$-semigroups). Let $0 \neq S \in \mathscr{L} \mathscr{M}=\mathscr{M}_{2}$. By definition $S$ contains non-zero $\mathscr{M}$-ideals. Let us denote by $I_{1}$ the union of all $\mathscr{M}$-ideals of $S$. Since $\mathscr{M}$ consists of 0 -simple and one-element semigroups, so clearly $I_{1}$ is the 0 -disjoint union of $\mathscr{M}$-ideals of $S$. Define the ideals $I_{\beta}$ of $S$ as follows.

(i) If $\beta-1$ exists, then let $I_{\beta} / I_{\beta-1}$ be the union of all $\mathscr{M}$-ideals of $S / I_{\beta-1}$.

(ii) If $\beta$ is a limit ordinal, then let $I_{\beta}$ be the union of all ideals $I_{\alpha}, \alpha<\beta$.

This process will terminate at an ordinal number $\zeta$. We assert $I_{\zeta}=S$. If $I_{\zeta} \neq S$, then $S / I_{\zeta} \neq 0$ contains a non-zero $\mathscr{M}$-ideal, and so $I_{\zeta+1}$ contains properly $I_{\zeta}$ which is a contradiction.

Conversely, suppose that to $S$ there exist an ordinal number $\zeta$ and a strictly ascending chain

$$
0=I_{0} \subset I_{1} \subset \ldots \subset I_{\lambda} \subset \ldots \subset I_{\zeta}=S
$$

of ideals of $S$ such that $I_{\lambda+1} / I_{\lambda}$ is the disjoint union of $\mathscr{M}$-ideals of $S / I_{\lambda}(0 \leqq \lambda<\zeta)$, and $I_{\lambda}=\bigcup_{\mu<\alpha} I_{\mu}$ if $\lambda$ is a limit ordinal. Let $J \neq S$ be an arbitrary ideal of $S$ and $S^{\prime}=S / J$ the Rees factor semigroup. Consider the ordinal number $v$ such that $J_{\mu} \subseteq J$ for all $\mu<v, \mu \geqq 0$ and $I_{v} \ddagger J$. Obviously such a $v$ does exist and it must not be a limit ordinal. Since $I_{v-1} \subseteq I_{v} \cap J$, therefore $I_{v} /\left(I_{v} \cap J\right)$ is a Rees factor semigroup of $I_{v} / I_{v-1}$. By the assumption $I_{v} / I_{v-1}$ is the union of $\mathscr{U}$-ideals of $S / I_{v-1}$. By the second isomorphism theorem we have $I_{v} /\left(I_{v} \cap J\right) \cong\left(I_{v} \cup J\right) / J$, and so there exists a non-zero $\mathscr{U}$-ideal $M / J$ of $\left(I_{v} \cup J\right) / J$ and by Proposition $4 M / J$ is an ideal of $S / J$. 
Since the choise of $J$ was arbitrary, therefore $S \in \mathscr{M}_{2}=\mathscr{L} \mathscr{M}$ follows. Thus taking into account Theorem 1 we have obtained

Theorem 2. Let $\mathscr{M}$ be a class of 0 -simple idemoptent semigroups such that $0 \in \mathscr{M}$. Then the following conditions are equivalent.

(1) The semigroup $S$ is an $\mathscr{L} \mathscr{M}$-radical semigroup.

(2) Every non-zero Rees factor semigroup of the semigroup $S$ contains a non-zero $\mathscr{M}$-ideal.

(3) There exist am ordinal number $\zeta$ depending on $S$ and a strictly ascending chain

$$
0=I_{0} \subset I_{1} \subset \ldots \subset I_{\lambda} \subset \ldots \subset I_{\zeta}=S
$$

of ideals of $S$ such that for each $0 \leqq \lambda<\zeta$ the Rees factor semigroup $I_{\lambda+1} / I_{\lambda}$ is the 0 -disjoint union of $\mathscr{M}$-ideals of $S / I_{\lambda}, 0 \leqq \lambda<\zeta$ and $I_{\lambda}=\bigcup_{\mu<\lambda} I_{\mu}$ if $\lambda$ is a limit
ordinal.

The case when $\mathscr{M}$ consists of all completely 0 -simple semigroups, is of special interest, because the structure of complete 0-simple semigroups and of their disjoint unions has been determined in the papers of ReEs [5] and STEINFELD [7].

\section{References}

[1] E. P. Armendariz - W. G. Leavitt: The hereditary property in the lower radical construction, Can. J. Math., 20 (1968), 474-476.

[2] A. H. Clifford-G. B. Preston: The algebraic theory of emigroups I-II., Providence, 1961 and 1967.

[3] N. Divinsky: Rings and radicals, London, 1965.

[4] Е. Ц. Ллпин: Полугруппы. Москва. 1961,

[5] D. Rees: On semigroups, Proc. Cambridge Philos. Soc., 36 (1940), 387-400.

[6] Št. Schwarz: O nолугрупnах, имеющих ядро, Czechoslovak Math., J., 1 (76) (1951), 229-264.

[7] $O$. Steinfeld: On semigroups which are unions of completely 0 -simple subsemigroups, Czechoslovak Math. J., 16 (91) (1966), 63-69.

[8] O. Steinfeld: On a generalization of completely 0- simple semigroups, Acta Sci. Math., 28 (1967), 135-145.

[9] A. Suliński - R. Anderson-N. Divinsky: Lowel radical properties for associative and alternative rings, J. London: Math. Soc., 41 (1966), 417-424.

[10] F. Szász-R. Wiegandt: On the duality of radical and semisimple objects in categories, Acta Math. Acad. Sci. Hung., 21 (1970), 175-182.

[11] J. F. Watters: Lower radicals in associative rings, Can J. Math., 21 (1969), 466-476.

[12] R. Wiegandt: Structure theorems for objects, Publ. Math. (Debrecen), (to appear).

Author's address: Mathematical Institute of the Hungarian Academy of Sciences, Reáltanoda u. 13/15 Budapest, V. Hungary. 